\title{
Biomarkers of Habitable Worlds - Super-Earths and Earths
}

\author{
L. Kaltenegger ${ }^{12}$ \\ ${ }^{1}$ MPIA, Koenigstuhl 17, 69117 Heidelberg, Germany \\ email: kaltenegger@mpia.de \\ ${ }^{2}$ CfA, 60 Garden street, Cambridge, 02138 MA, USA
}

\begin{abstract}
A decade of exoplanet search has led to surprising discoveries, from giant planets close to their star, to planets orbiting two stars, all the way to the first extremely hot, rocky worlds with potentially permanent lava on their surfaces due to the star's proximity. Observation techniques have reached the sensitivity to explore the chemical composition of the atmospheres as well as physical structure of some detected planets. Recent advances in detection techniques find planets of less than $10 M_{\text {Earth }}$ (so called Super-Earths), among them some that may potentially be habitable. Two confirmed non-transiting planets and several transiting Kepler planetary candidates orbit in the Habitable Zone of their host star. The detection and characterization of rocky and potentially Earth-like planets is approaching rapidly with future ground- and spacemissions, that can explore the planetary environments by analyzing their atmosphere remotely. The results of a first generation space mission will most likely be an amazing scope of diverse planets that will set planet formation, evolution as well as our planet in an overall context.
\end{abstract}

Keywords. Earth, atmospheric effects, astrobiology

\section{Introduction}

The current status of exoplanet characterization shows a surprisingly diverse set of giant planets. For a subset of these, some properties have been measured or inferred using radial velocity (RV), micro-lensing, transits, and astrometry. These observations have yielded measurements of planetary mass, orbital elements and (for transits) the planetary radius and during the last few years, physical and chemical characteristics of the upper atmosphere of some of the transiting planets (see e.g. a review by (Seager \& Deming 2010) and references therein). Specifically, observations of transits, combined with RV information, have provided estimates of the mass, radius and density of a subset of these planets, ranging from Giant planets to rocky planets like Corot 7b (Léger et al. 2009) and Kepler 10b (Batalha et al. 2011). Most detected planets orbit extemely close to their host star due to a detection bias and therefore receive high amounts of stellar irradiation and have subsequent high surface temperature. Recent investigations of high precision radial velocity data samples have shown that between $20 \%$ and $50 \%$ of all sample stars exhibit RV variations indicating the presence of super-Earths or ice giants (Lovis et al. 2009), (Howard et al. 2011). Among the hundreds of confirmed planets, already two close-by, low mass RV planets, Gl 581 d (Udry et al. 2007), (Mayor et al. 2009) and HD 85512 b (Pepe et al. 2011), with minimum masses of 7 and 3.6 Earth masses, respectively, orbit in the Habitable Zone of their parent star on the outer and inner edge respectively. These close-by planets provide excellent targets for future atmospheric exploration. Several Kepler transit planetary candidates from the February 2011 data release (Borucki et al. 2011), that are consistent with rocky models, orbit their host stars 
also in the Habitable Zone, providing first statistics of the number of planets and eta Earth $_{\text {a }}$ (see e.g. (Traub 2011)) and a more complete sample is expected in the second Kepler data release in 2012. The discovery of transiting planets with masses below 10 Earth masses and radii consistent with rocky planetary models, answered the important question if planets more massive than Earth could potentially be rocky. 10 Earth masses are used from formation theories as the upper limit for rocky planet formation, for comparison, Uranus has about 15 Earth masses. Above that mass the planet is thought to accumulate a substantial amount of gas that makes it akin to a gas giant, not a rocky planet with an outgassed atmosphere. Where exactly such a cut-off mass is - if it exists at all - is an open question. Recent discoveries by ground based, as well as the Corot and Kepler mission, found planets with masses below 10 Earth masses and densities akin to Neptune as well as Earth, suggesting that there is not one cut-off mass above which a planet is like Neptune and below which it is rocky like Earth or Venus. The first planets below $10 M_{\text {Earth }}$, with both mass estimates and radius measurements, have provided a wide range of observed radii and densities. Especially in the mass range below $5 M_{E a r t h}$, two planets in the multiple planet system, Kepler 11b and Kepler 11f (Lissauer et al. 2011), with 4.3 and $2.3 M_{\text {Earth }}$ have radii of 1.97 and 2.61 Earth radii and mean densities of 3.1 and $0.7 \mathrm{~g} / \mathrm{cm}^{3}$, respectively. These derived densities also allow substantial envelopes of light gases for this mass range. Recent atmosphere observations for a $6.55 \pm 0.98$ Earth mass planet, GJ $1214 \mathrm{~b}$, with a mean density of $1.8 \mathrm{~g} / \mathrm{cm}^{3}$ (Charbonneau et al. 2009) (for atmosphere observations see e.g. (Bean et al. 2010), (Désert et al. 2011)), indicates either hazes or high cloud cover in an expanded atmosphere.

Observing mass and radius alone can not break the degeneracy of a planet's nature due to the effect of an extended atmosphere that can also block the stellar light and increase the observed planetary radius significantly (see e.g. (Adams et al. 2008)). Even if a unique solution would exist, planets with similar density, like Earth and Venus, present very different planetary environments in terms of habitable conditions. Therefore the question refocusses on atmospheric features to characterize a planetary environment. The atmosphere is the only accessible quantity that allows exploring a planetary environment remotely.

\section{The Habitable Zone}

Different aspects of what determines the boundaries of the HZ have been discussed broadly in the literature. The main differences among these studies are the climatic constraints imposed. Here we focus on the circumstellar HZ defined by J. Kasting in 1993 (Kasting et al. 1993) as an annulus around a star where a planet with a $\mathrm{CO}_{2} / \mathrm{H}_{2} \mathrm{O} / \mathrm{N}_{2}$ (Earth-like composition) atmosphere, surface pressures between 1 and 10 bar and a sufficiently large water content, like Earth, can host liquid water permanently on a solid surface. In this definition, the two edges of the HZ (see Fig. 1) as well as the equilibrium temperature of the planet, $T_{e q}$, depend on the Bond albedo of the planet $A$, the luminosity of the star $L_{\text {star }}$, the planet's semi major axis $D$, as well as the eccentricity $e$, of the orbit, and in turn the average stellar irradiation at the planet's location (see also (Selsis et al. 2007)). Note that this definition of the HZ implies surface habitability and in turn allows remote detectability of life as we know it. Subsurface life that could exist on planets with very different surface temperatures is not considered here, because of the lack of remotely detectable atmospheric to assert habitability.

A more eccentric orbit increases the annually averaged irradiation proportional to (1$\left.e^{2}\right)^{-1 / 2}$ (see (Williams \& Pollard 2002)). The inner edge of the HZ denotes the location 


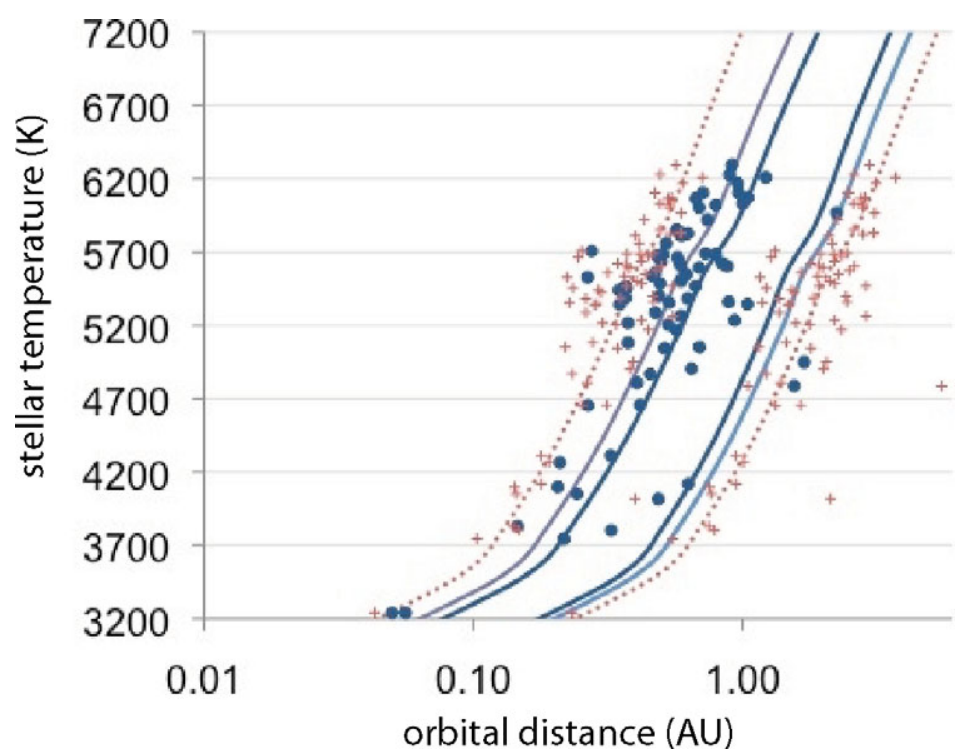

Figure 1. Extent of the HZ for water loss limit for $0 \%$ and $50 \%$ cloud coverage (inner limits) and $100 \%$ cloud coverage (outer limit dashed line) and position of potentially habitable Kepler planetary candidates in the HZ, individual HZ limits are indicated with crosses (Kaltenegger \& Sasselov 2011).

where the entire water reservoir can be vaporized by runaway greenhouse conditions, followed by the photo-dissociation of water vapor and subsequent escape of free hydrogen into space. The outer boundary denotes the distance from the star where the maximum greenhouse effect fails to keep $\mathrm{CO}_{2}$ from condensing permanently, leading to runaway glaciation. Note that at the limits of the HZ, the Bond albedo of a habitable planet is fully determined by its atmospheric composition and de- pends on the spectral distribution of the stellar irradiation. To simply estimate if an Earth-like planet as defined above is potentially habitable $\left(175 K<T_{e q}<270 K\right)$ (see e.g. (Kaltenegger et al. 2011a)), one can approximate $T_{e q}$ for Earth-like planets around different stars using the maximum albedo for rocky planet atmospheres, that varies depending on the planet's cloud cover as well as host star. Applying this definition to the Kepler Feb 2011 data release, assuming circular orbits and albedo corresponding to $50 \%$ cloud coverage (that is also consistent with the empirical "Venus"-limit of the HZ), leads to 27 Kepler planetary candidates with $175 K<T_{e q}<270 K$ (see Fig. 1). Among those are 3 planetary candidates that have radii smaller than 2 Earth radii (Kaltenegger \& Sasselov 2011). The potentially rocky planet candidates in multiple systems (see e.g. (Lissauer et al. 2011)) are extremely interesting objects because their mass could be determined using transit time variations to calculate a mean density and potentially confirm high density and rocky characteristics.

The HARPS Radial Velocity program recently published the RV data and the orbital parameters of a new low-mass planet of $3.6 \pm 0.5 M_{\text {Earth }}$ min. mass around the star HD 85512 (Pepe et al. 2011). HD 85512 is a K5V star with $0.126 \pm 0.008 L_{\text {Sun }}, 0.69$ $M_{\text {Sun }}$ and an effective temperature, $T_{\text {eff }}$, of $4715 \pm 102$. HD $85512 \mathrm{~b}$ orbits its star in $58.43 \pm 0.13$ days at $0.26 \pm 0.005$ AU with an eccentricity of $0.11 \pm 0.1$, which places it inside the and near to the inner edge of the Habitable Zone (HZ). The planet is one of the least massive planets detected to date. It is the least massive planet confirmed in the HZ. Low mass Main Sequence M dwarfs are the most abundant stars in the 


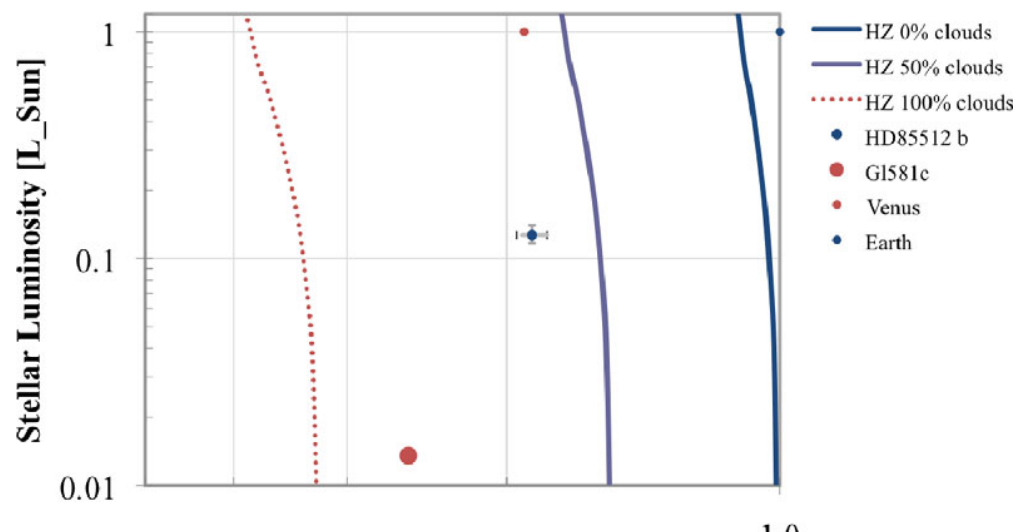

Stellar Flux [S_0]

Figure 2. $L_{\text {star }}$ in $L_{S u n}$, versus stellar flux on top of the planetary atmosphere in $S_{0}$. The extent of the inner edge of the HZ for the water loss limit for $0 \%, 50 \%$ and $100 \%$ (dashed line) cloud coverage (from right to left) and Earth, Venus and Gl 581c are added for comparison (Kaltenegger et al. 2011b) . Note that the x-axis decreases towards the right.

galaxy, representing about $75 \%$ of the total stellar population. Many planets including potentially rocky planets within the Habitable Zone (HZ) like Gl581d (Selsis et al. 2007), (von Bloh et al. 2007), (von Paris et al. 2011),(Wordsworth et al. 2011), (Kaltenegger et al. 2011a), (Hu \& Ding 2011)) are thus likely to be found in the near future, providing excellent targets ((Scalo et al. 2007), (Tarter et al. 2007), (Kaltenegger et al. 2010a)), that can be probed for atmospheric components, especially for hot planets with extended atmospheres.

Fig. 2 shows the position and borders of the HZ. For comparison the values for Earth and Venus as well as the exoplanet Gl 581c are shown.

Several designs for future space missions that have the explicit purpose of detecting other Earth-like worlds as well as analyzing their characteristics, determining the composition of their atmospheres, and searching for signs of life are under study at ESA as well as NASA. They also have the capacity to investigate the physical properties and composition of a broader diversity of planets, to understand the formation of planets and some even have the capability to interpret potential biosignatures.

\section{A SPECTRAL FINGERPRINT OF AN ATMOSPHERE}

A planet is a very faint, small object close to a very bright and large object, its parent star. In the visible part of the spectrum we observe the starlight, reflected off the planet, in the IR we detect the planets own emitted flux. The Earth-Sun intensity ratio is about $10^{-7}$ in the thermal infrared $(10 \mu \mathrm{m})$, and about $10^{-10}$ in the visible $(0.5 \mu \mathrm{m})$ (see Fig. 3). Nevertheless the contrast ratio of hot extrasolar Giant planets (EGP) to their parent stars flux as well as the contrast ratio of a planet to a smaller parent star is much more favorable, making Earth-like planets around small stars very interesting targets (see e.g. (Scalo et al. 2007), (Tarter et al. 2007)).

Different strategies exist to characterize a planet's atmosphere: direct detection that resolves the planet and star individually and transmission as well as secondary eclipse measurements that subtract the stellar light from a combined star-planet detection. The 
first imaged exoplanet candidates around young stars show the improvement in direct detection techniques that are designed to resolve the planet and collect its photons. This can currently be achieved for widely separated young objects and has already detected exoplanets candidates (see e.g. (Kalas et al. 2008), (Lagrange et al. 2010), (Marois et al. 2008)). Future telescopes will allow for direct detection in even closer orbits for close-by stars. In the next years ground-based as well as space missions will give us statistics on the number, size, period and orbital distance of planets, extending to terrestrial planets on the lower mass range end as a first step, while future space missions are designed to characterize their atmospheres.

The atmosphere of a planet contains the detectable information to explore the planetary environment remotely. The spectrum of a planet can contain signatures of atmospheric species, what creates its spectral fingerprint. On Earth some atmospheric species exhibiting noticeable spectral features in the planet's spectrum result directly or indirectly from biological activity: the main ones are $\mathrm{O}_{2}, \mathrm{O}_{3}, \mathrm{CH}_{4}$, and $\mathrm{N}_{2} \mathrm{O}$ (see Fig. 3 and (Kaltenegger \& Traub 2009), (Rauer et al. 2011) (Pallé et al. 2009)). $\mathrm{CO}_{2}$ and $\mathrm{H}_{2} \mathrm{O}$ are in addition important as greenhouse gases in a planet's atmosphere and potential sources for high $\mathrm{O}_{2}$ concentration from photosynthesis (see e.g. (Des Marais et al. 2002), (Kaltenegger et al. 2010c). Note that similar features could be detectable on Earth-like moons (Williams et al. 1997) (Kaltenegger 2010).

Sagan et al. (Sagan et al. 1993) analyzed a spectrum of the Earth taken by the Galileo probe in 1993, searching for signatures of life and concluded that the large amount of $\mathrm{O}_{2}$ and the simultaneous presence of $\mathrm{CH}_{4}$ traces are strongly suggestive of biology for a planet around a Sun-like star. Fig. 3 shows the detectable features in the planet's reflection, emission and transmission spectrum using the Earth itself as a proxy for observations and model fits to data of spectra of the Earth.

Our search for signs of life is based on the assumption that extraterrestrial life shares fundamental characteristics with life on Earth, in that it requires liquid water as a solvent and has a carbon-based chemistry (see e.g. (Brack 1993), (Owen 1980). Life on the
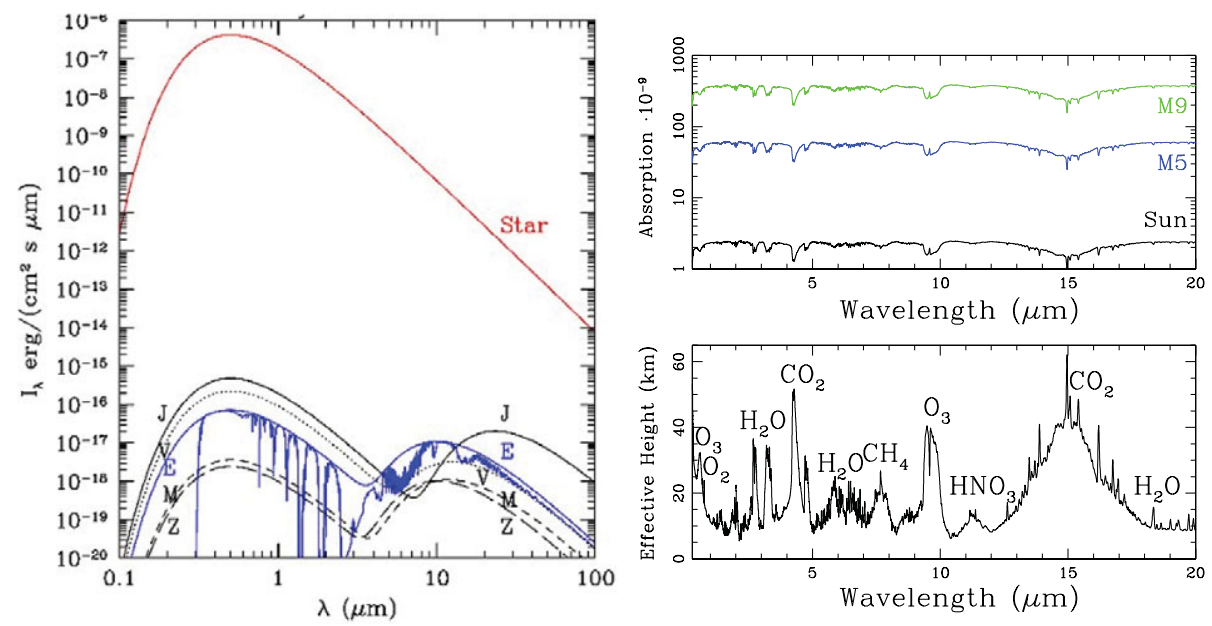

Figure 3. Model of our Solar System (left) (assumed here to be Black Bodies with Earth spectrum shown). Synthetic transmission spectra (right) of the Earth from UV to IR shown. The intensity is given as a fraction of solar intensity as well as the relative height in the atmosphere. The atmospheric features are indicated (figure adapted from (Kaltenegger et al. 2010c) and (Kaltenegger \& Traub 2009)) 
base of a different chemistry is not considered here because the vast possible life-forms produce signatures in their atmosphere that are so far unknown. Therefore we assume that extraterrestrial life is similar to life on Earth in its use of the same input and output gases, that it exists out of thermodynamic equilibrium (see e.g. (Lovelock 1975) (Rosenqvist \& Chassefiere 1995)). Biomarkers is used here to mean detectable species, or set of species, whose presence at significant abundance strongly suggests a biological origin (e.g. couple $\mathrm{CH}_{4}+\mathrm{O}_{2}$, or $\mathrm{CH}_{4}+\mathrm{O}_{3}$ ). Bio-indicators are indicative of biological processes but can also be produced abiotically. It is their quantities, and detection along with other atmospheric species, in a certain context (for instance the properties of the star and the planet) that points toward a biological origin.

It is relatively straightforward to remotely ascertain that Earth is a habitable planet, replete with oceans, a greenhouse atmosphere, global geochemical cycles, and life - if one has data with arbitrarily high signal-to-noise and spatial and spectral resolution. The interpretation of observations of other planets with limited signal-to-noise ratio and spectral resolution as well as absolutely no spatial resolution, as envisioned for the first generation instruments, will be far more challenging and implies that we need to gather information on the planet environment to understand what we will see (see e.g. (Kaltenegger et al. 2010c)): After detection, we will focus on main properties of the planetary system, its orbital elements as well as the presence of an atmosphere using the light curve of the planet or/and a crude estimate of the planetary nature using very low-resolution information (3 or 4 channels).

Presently, radius measurements can only be performed when the planet transits in front of its parent star, by an accurate photometric technique. If the secondary eclipse of the transiting planet can be observed (when the planet passes behind the star), then the thermal emission of the planet can be measured, allowing the retrieval of mean brightness temperature thanks to the knowledge of the radius from the primary transit. If a nontransiting target is observed in both visible and IR ranges, the albedo can be estimated in the visible once the radius is inferred from the IR spectrum, and compared with one derived from the thermal emission only.

Then a higher resolution spectrum will be used to identify the compounds of the planetary atmosphere, constrain the temperature and radius of the observed exoplanet. In that context, we can test if we have an abiotic explanation of all compounds seen in the atmosphere of such a planet. If we do not, we can work with the exciting biotic hypothesis. $\mathrm{O}_{2}, \mathrm{O}_{3}, \mathrm{CH}_{4}$ are good biomarker candidates that can be detected by a low-resolution (Resolution $<80$ ) spectrograph. Note that the presence of biogenic gases such as $\mathrm{O}_{2} / \mathrm{O}_{3}+\mathrm{CH}_{4}$ may imply the presence of a massive and active biosphere, but their absence does not imply the absence of life. Life existed on Earth before the interplay between oxygenic photosynthesis and carbon cycling produced an oxygen-rich atmosphere (see Fig. 4).

The coronagraph and occulter concepts detect the reflected light of a planet and operate in the visible and near infrared $(0.5-1 \mu \mathrm{m})$ with a minimum resolution of 80 . The interferometric systems suggested for future direct imaging missions operate in the mid-IR (6-20 $\mu \mathrm{m})$ and observe the thermal emission emanating from the planet with a minimum resolution of 25 . The viewing geometry results in different flux contribution of the overall detected signal from the bright and dark side, for the reflected light, and the planet's hot and cold regions for the emitted flux. Both spectral regions contain the signature of atmospheric gases that can be observed with low resolution and can indicate habitable conditions and, possibly, the presence of a biosphere: $\mathrm{CO}_{2}, \mathrm{H}_{2} \mathrm{O}, \mathrm{O}_{3}, \mathrm{CH}_{4}$, and $\mathrm{N}_{2} \mathrm{O}$ in the thermal infrared, and $\mathrm{H}_{2} \mathrm{O}, \mathrm{O}_{3}, \mathrm{O}_{2}, \mathrm{CH}_{4}$ and $\mathrm{CO}_{2}$ in the visible to near-infrared. The presence or absence of these spectral features (detected collectively) will indicate 
similarities or differences with the atmospheres of terrestrial planets, and its astrobiological potential.

\section{Evolution of biomarkers over geological times on Earth}

One crucial factor in interpreting planetary spectra is the point in the evolution of the atmosphere when its biomarkers and its habitability become detectable. The spectrum of the Earth has not been static throughout the past 4.5 billion years ago $(\mathrm{Ga})$. This is due to the variations in the molecular abundances, the temperature structure, and the surface morphology over time. At about 2.3 Ga oxygen and ozone became abundant, affecting the atmospheric absorption component of the spectrum. At about $0.44 \mathrm{Ga}$, an extensive land plant cover followed, generating the red chlorophyll edge in the reflection spectrum. The composition of the surface (especially in the visible), the atmospheric composition, and temperature-pressure profile can all have a significant influence on the detectabilty of a signal.

Fig. 4 shows theoretical visible and mid-infrared spectra of the Earth over the main six epochs during its geological evolution. The epochs are chosen to represent major developmental stages of the Earth, and life on Earth. If an extrasolar planet is found with a corresponding spectrum, we can use the stages of evolution of our planet to characterizing it, in terms of habitability and the degree to which it shows signs of life, assuming similar evolution time scales. Furthermore we can learn about the evolution of our own planet's atmosphere and possible the emergence of life by observing exoplanets in different stages of their evolution. Earth's atmosphere has experienced dramatic evolution over 4.5 billion years, and other planets may exhibit similar or greater evolution, and at different rates. It shows epochs that reflect significant changes in the chemical composition of the atmosphere. The ozone/oxygen absorption features could have been used to indicate the presence of biological activity on Earth in combination with a reducing gas like $\mathrm{CH}_{4}$ anytime during the past $50 \%$ of the age of the solar system. Different signatures in the atmosphere are clearly detectable over Earth's evolution and observable with low resolution.
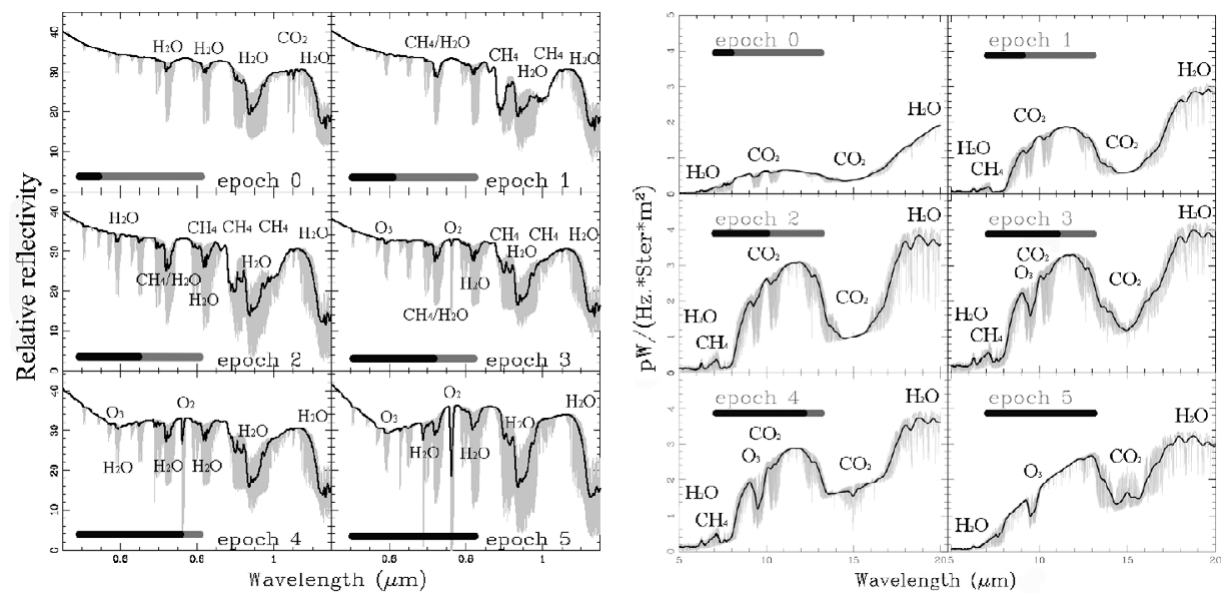

Figure 4. The visible to near-IR (left) and mid IR (right) spectral features on an Earth-like planet change considerably over its evolution from a $\mathrm{CO}_{2}$ rich (epoch 0) to a $\mathrm{CO}_{2} / \mathrm{CH}_{4}$-rich atmosphere (epoch 3) to a present-day atmosphere (epoch 5). The bold lines show spectral resolution of 80 and 25 comparable to the proposed visible TPF-C/New World Observer and Darwin/TPF-I mission concept, respectively. 

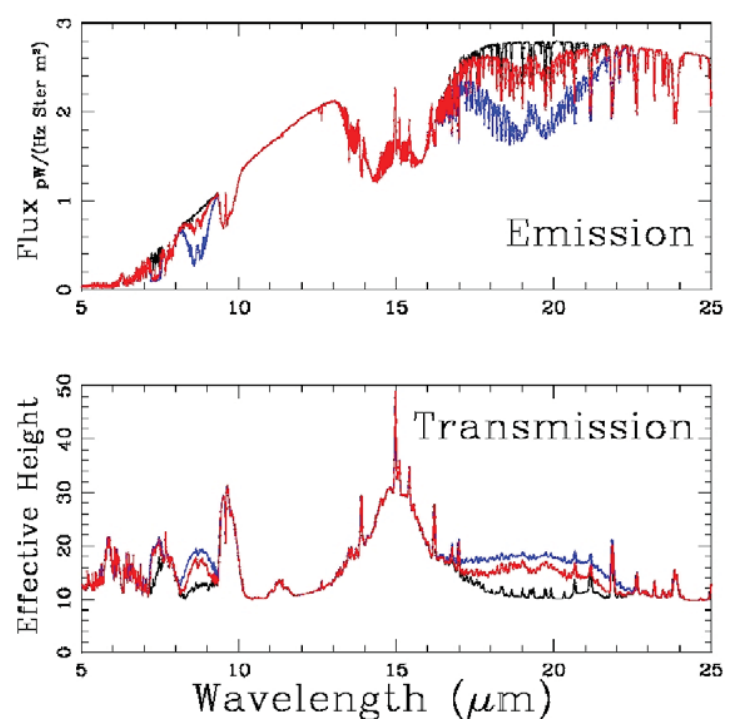

Figure 5. Current Earth spectra model: emergent (top) and transmission (bottom) spectra 5 $\mu \mathrm{m}$ to $25 \mu \mathrm{m}$ of an Earth atmosphere including water clouds at $1 \mathrm{~km}, 5 \mathrm{~km}$ and $12 \mathrm{~km}$ for three volcanic $\mathrm{SO}_{2}$ concentrations (Black: No eruption. Red: 10x Pinatubo eruption (baseline). Blue: $100 \mathrm{x}$ baseline). The calculations are performed at very high resolution ( 0.1 wavenumbers) and subsequently smoothed for display $(\mathrm{R}=150)$ (adapted from (Kaltenegger et al. 2010b)).

Such spectra will be used as part of a big grid to characterize any exoplanets found and influences the design requirements for a spectrometer to detect habitable planets, a grid different international groups are working on (see e.g. (Rauer et al. 2011), (Segura et al. 2003), (Spiegel et al. 2010), (Forget \& Pierrehumbert 1997), (Cowan et al. 2009), (Goldblatt \& Zahnle 2011), (Edson et al. 2011), (Kitzmann et al. 2010), (Domagal-Goldman et al. 2011),(Kaltenegger et al. 2010c) and references therein). Other geochemical cycles as well as volcanic events can also leave observable features in a planet's atmosphere that can potentially be detectable (see Fig. 5) (see also e.g. (Kaltenegger et al. 2010b)).

\section{Summary}

Spectroscopy of the atmosphere of extrasolar planets allows to remotely explore a planet's environment, distinguishing Mini-Neptunes from rocky Super-Earths, exploring atmospheric compositions as well as searching for indications for habitability. Any information we collect on habitability is only important in a context that allows us to interpret, what we find. To search for signs of life we need to understand how the observed atmosphere physically and chemically works. Knowledge of the temperature and planetary radius is crucial for the general understanding of the physical and chemical processes occurring on the planet. These parameters as well as an indication of habitability can be determined with low resolution spectroscopy and low photon flux, as assumed for first generation space missions. The combination of spectral information in the visible (starlight reflected off the planet) as well as in the mid-IR (planet's thermal emission) allows a confirmation of atmospheric species, a more detailed characterization of individual planets but also to explore a wide domain of planet diversity. Being able to measure the outgoing shortwave and longwave radiation as well as their variations along 
the orbit, to determine the albedo and identify greenhouse gases, would in combination allow us to explore the climate system at work on the observed worlds, as well as probe planets similar to our own for habitable conditions.

\section{Acknowledgement}

L.K. acknowledges support from NAI and DFG funding ENP Ka 3142/1-1.

\section{References}

Adams, E. R., Seager, S., \& Elkins-Tanton, L. 2008, ApJ, 673, 1160

Batalha, N. M., Borucki, W. J., Bryson, S. T., et al. 2011, ApJ, 729, 27

Bean, J. L., Miller-Ricci Kempton, E., \& Homeier, D. 2010, Nature, 468, 669

Borucki, W. J., Koch, D. G., Basri, G., et al. 2011, ApJ, 736, 19

Brack, A. 1993, Origins of Life and Evolution of the Biosphere, 23, 3

Charbonneau, D., Berta, Z. K., Irwin, J., et al. 2009, Nature, 462, 891

Cowan, N. B., Agol, E., Meadows, V. S., et al. 2009, ApJ, 700, 915

Des Marais, D. J., Harwit, M. O., Jucks, K. W., et al. 2002, Astrobiology, 2, 153

Désert, J.-M., Bean, J., Miller-Ricci Kempton, E., et al. 2011, ApJ, 731, L40

Domagal-Goldman, S. D., Meadows, V. S., Claire, M. W., \& Kasting, J. F. 2011, Astrobiology, 11,419

Edson, A., Lee, S., Bannon, P., Kasting, J. F., \& Pollard, D. 2011, Icarus, 212, 1

Forget, F. \& Pierrehumbert, R. T. 1997, Science, 278, 1273

Goldblatt, C. \& Zahnle, K. J. 2011, Nature, 474

Howard, A. W., Marcy, G. W., Bryson, S. T., et al. 2011, ArXiv e-prints

Hu, Y. \& Ding, F. 2011, A\&SA, 526, A135

Kalas, P., Graham, J. R., Chiang, E., et al. 2008, Science, 322, 1345

Kaltenegger, L. 2010, ApJ, 712, L125

Kaltenegger, L., Eiroa, C., Ribas, I., et al. 2010a, Astrobiology, 10, 103

Kaltenegger, L., Henning, W. G., \& Sasselov, D. D. 2010b, AJ, 140, 1370

Kaltenegger, L. \& Sasselov, D. 2011, ApJ, 736, L25

Kaltenegger, L., Segura, A., \& Mohanty, S. 2011a, ApJ, 733, 35

Kaltenegger, L., Selsis, F., Fridlund, M., et al. 2010c, Astrobiology, 10, 89

Kaltenegger, L. \& Traub, W. A. 2009, ApJ, 698, 519

Kaltenegger, L., Udry, S., \& Pepe, F. 2011b, ArXiv e-prints

Kasting, J. F., Whitmire, D. P., \& Reynolds, R. T. 1993, Icarus, 101, 108

Kitzmann, D., Patzer, A. B. C., von Paris, P., et al. 2010, A\& A, 511, A66

Lagrange, A.-M., Bonnefoy, M., Chauvin, G., et al. 2010, Science, 329, 57

Léger, A., Rouan, D., Schneider, J., et al. 2009, A\&A, 506, 287

Lissauer, J. J., Fabrycky, D. C., Ford, E. B., et al. 2011, Nature, 470, 53

Lovelock, J. E. 1975, Royal Society of London Proceedings Series B, 189, 167

Lovis, C., Mayor, M., Bouchy, F., et al. 2009, in IAU Symposium, Vol. 253, IAU Symposium, $502-505$

Marois, C., Macintosh, B., Barman, T., et al. 2008, Science, 322, 1348

Mayor, M., Bonfils, X., Forveille, T., et al. 2009, A\&A, 507, 487

Owen, T. 1980, in Astrophysics and Space Science Library, Vol. 83, Strategies for the Search for Life in the Universe, ed. M. D. Papagiannis, 177

Pallé, E., Zapatero Osorio, M. R., Barrena, R., Montañés-Rodríguez, P., \& Martín, E. L. 2009, Nature, 459, 814

Pepe, F., Lovis, C., Ségransan, D., et al. 2011, ArXiv e-prints

Rauer, H., Gebauer, S., Paris, P. V., et al. 2011, A\&A A, 529, A8

Rosenqvist, J. \& Chassefiere, E. 1995, 43, 3 
Sagan, C., Thompson, W. R., Carlson, R., Gurnett, D., \& Hord, C. 1993, 365, 715

Scalo, J., Kaltenegger, L., Segura, A. G., et al. 2007, Astrobiology, 7, 85

Seager, S. \& Deming, D. 2010, ARA\& A, 48, 631

Segura, A., Krelove, K., Kasting, J. F., et al. 2003, Astrobiology, 3, 689

Selsis, F., Kasting, J. F., Levrard, B., et al. 2007, A\&A, 476, 1373

Spiegel, D. S., Raymond, S. N., Dressing, C. D., Scharf, C. A., \& Mitchell, J. L. 2010, ApJ, 721, 1308

Tarter, J. C., Backus, P. R., Mancinelli, R. L., et al. 2007, Astrobiology, 7, 30

Traub, W. A. 2011, ArXiv e-prints

Udry, S., Bonfils, X., Delfosse, X., et al. 2007, A\& $A$, 469, L43

von Bloh, W., Bounama, C., Cuntz, M., \& Franck, S. 2007, AESA, 476, 1365

von Paris, P., Gebauer, S., Godolt, M., Rauer, H., \& Stracke, B. 2011, A\& A, 532, A58

Williams, D. M., Kasting, J. F., \& Wade, R. A. 1997, Nature, 385, 234

Williams, D. M. \& Pollard, D. 2002, International Journal of Astrobiology, 1, 61

Wordsworth, R. D., Forget, F., Selsis, F., et al. 2011, ApJ, 733, L48

\section{Discussion}

MELNICK: How doe modest changes in eccentricity affect the atmosphere of Super-Earth and Earth-like planets?

KALTENEGGER: The effect on the spectrum of an Earth-like atmosphere would be small if the eccentricity is moderate, see e.g. the work by (Williams \& Pollard 2002). If the eccentricity is high, that can have an influence on the planet's climate see e.g. (Spiegel et al. 2010) and therefore also show detectable features in its spectrum. It depends on how dense the atmosphere is and what the heating \& cooling times of the atmosphere are in comparison to the time the planet spends close or far away from its star due to eccentricity.

ENCRENAZ: On two occasions the Earth almost froze over.Volcanoes warmed up the crust, probably due to greenhouse effects generated by $\mathrm{SO}_{2}$. We should also investigate this molecule in exoplanets.

KALtenegGer: There are different opinions what warmed the atmosphere and got us out of Earth's snowball phase(s). The main contenders now are the greenhouse effect generated by $\mathrm{CO}_{2}$ and/or as you mentioned by $\mathrm{SO}_{2}$. We explored (Kaltenegger et al. $2010 \mathrm{~b}$ ) if you could potentially detect $\mathrm{SO}_{2}$ and in turn volcanic activity on exoplanets, or if you want to turn the question around, what the minimum amount of $\mathrm{SO}_{2}$ in Earth's current and Archean atmosphere is, to be detectable with low resolution (see Fig. 4). We found that 10 to 100 times a Pinatubo eruption would leave a remotely detectable $\mathrm{SO}_{2}$ feature in the infrared spectrum in both emission and transmission. That is very interesting because it would allow us to test whether volcanism is a common occurrence on rocky exoplanets, and if it - and underlying concepts like tectonic or a planetary liquid interior - are common and if they depend on the mass of the planet.

Regarding the snowball phase: One interesting point is that the climate on Earth and on a geological active rocky planet - is buffered by geological cycles, in our case the carbonate-silicate cycle, that regulates the concentration of $\mathrm{CO}_{2}$ in Earth's atmosphere. If the surface temperature gets colder, $\mathrm{CO}_{2}$ does not get rained out as efficiently of our atmosphere (and subsequently subducted), builds up and generates a stronger greenhouse effect that in turn warms the planet, generating liquid rainout and removal of excess 
$\mathrm{CO}_{2}$ from the atmosphere again. That is the underlying concept of why we think that the $\mathrm{CO}_{2}$ greenhouse effect helped de-freeze snowball Earth. Btw the carbonate-silicate cycle is also the reason why we have a Habitable Zone, not just a habitable Distance, because the feedback between surface temperature and $\mathrm{CO}_{2}$ concentration keeps the surface temperature of a rocky planet above freezing for a range of distances from it's star - as well as counters the initial low stellar flux Earth received. 